\title{
Characterization of peak shear strength of rough rock joints using limited displacement multi-stage direct shear (LDMDS) tests
}

\author{
Mary MacLaughlin ${ }^{1, *}$, Steve Berry ${ }^{1}$, Michael Petro ${ }^{2}$, Katherine Berry ${ }^{3}$, and Anders Bro ${ }^{4}$ \\ ${ }^{1}$ Montana Technological University, Department of Geological Engineering, 1300 West Park St, Butte, Montana, 59701 USA \\ ${ }^{2}$ Ransom Consulting Inc., Portsmouth, New Hampshire, 03801 USA \\ ${ }^{3}$ Freeport McMoRan, Safford, Arizona, 85546 USA \\ ${ }^{4}$ Swans Island, Maine, 04685 USA
}

\begin{abstract}
Current standard direct shear test methods for rock joints do not account for damage to the specimens' asperity profiles; tests require shearing of a single specimen to large displacements under successive normal stresses (the multistage test), or the use of similar specimens in multiple tests. Due to the inherently unique nature of rock joints and corresponding difficulty in obtaining specimens with identical or even similar geometries, multistage tests are more common. A major issue with the multistage test is that successive shearing of the specimen damages the surface asperities and changes its overall roughness profile, reducing the peak shear stress and consequently resulting in underestimation of the friction angle and overestimation of the joint shear intercept (cohesion). The limited displacement multistage direct shear (LDMDS) test method minimizes these testing imperfections by allowing shearing of a single specimen without extensive asperity damage, accomplished by immediately pausing shear displacement once peak shear stress has been reached, then proceeding to shear the specimen under the following normal stress value, and shearing into the post-peak region only after identifying multiple values of peak shear strength. The authors have validated the LDMDS procedure using cement replicates of rock joints, demonstrating that it yields more accurate strength parameters than the standard multistage direct shear test.
\end{abstract}

\section{Introduction}

The strength of jointed rock masses is controlled to a large degree by the relatively weak shear strengths of the discontinuity surfaces. Accurate determination of the shear strength of the discontinuities is critical for many rock engineering applications. The shear strength of rock joints is typically characterized using the standard MohrCoulomb parameters : joint friction angle and joint shear strength intercept (cohesion). These strength parameters are most often determined from direct shear tests conducted at a minimum of three different normal stress values according to ASTM and ISRM standards [1,2].

In contrast to soil materials, shear strength along a rough rock joint is a combination of the basic surficial strength and the additional shear resistance imparted by asperities on the joint surface. Since obtaining multiple test specimens with identical asperities is not possible due to the uniqueness of each rock joint surface, the standard direct shear test procedures for rock joints provide two alternatives : 1) shearing multiple similar specimens under different normal stresses, or 2) shearing a single unique specimen to large displacements multiple times under successive normal stresses. The second alternative, referred to as the multistage direct shear test, is much more popular because of the limited availability of similar specimens.
Theprimary problem with the multistage direct shear test is that specimens are sheared beyond the point of peak strength and the asperities on the specimen surface are increasingly damaged during each stage of shearing, resulting in lower shear strength. This has been recognized in previous studies [for example, 2-4] and in the current standards [1]. The inherent issue is that combining data from the first shear stage (performed on an 'undamaged' surface) with data from subsequent stages (on surfaces 'damaged' by previous shearing) results in underestimation of the peak friction angle and overestimation of the peak joint shear intercept [5].

To address this issue, an alternative procedure has been developed by Bro [6] which minimizes surface asperity damage by applying only extremely limited shear displacements until multiple values of peak shear strength have been achieved. This procedure is referred to as the Limited Displacement Multistage Direct Shear (LDMDS) test method. Although the issues with the current standard direct shear test procedure are recognized throughout the field of rock mechanics and others have developed test procedures similar to the LDMDS method [7], an improved test procedure has yet to be adopted.

Research has been undertaken at Montana Tech [8-10] to validate the LDMDS test method using replicates of rough rock joint surfaces cast out of synthetic materials. The results of the research, briefly summarized in this

\footnotetext{
* Corresponding author: mmaclaughlin@mtech.edu
} 
paper, also provide quantification of the degree of conservatism associated with the current standard multistage direct shear test procedure. The goal is to provide quantitative evidence that adjustments to the current test procedure can provide more accurate strength parameters.

\section{Direct shear test procedures}

The direct shear test is commonly used to characterize the shear strength of rock joints. A typical direct shear test is performed by encapsulating the two opposite surfaces of a specimen containing a discontinuity, applying a normal stress to the specimen, then displacing the specimen along the joint surface while recording the shear stress and the shear displacement. Normal displacement is usually recorded, as well, in order to quantify the dilation of the joint as the surfaces ride up and over the asperities.

In order to characterize the shear strength of a discontinuity, direct shear tests are commonly conducted at a minimum of three different normal stress values [1]. The peak shear stress values achieved under these three normal stress values are used to create a Mohr-Coulomb failure envelope from which the peak strength parameters of joint friction angle and joint shear intercept are determined by fitting a straight line through the data.

\section{1. 'Standard' multistage direct shear test}

As previously mentioned, the current 'standard' direct shear test procedure can be conducted in one of two ways: 1) testing multiple similar (but not identical) specimens, or 2 ) testing a single specimen multiple times $[1,2]$. Due to the difficulty of obtaining a sufficient number of similar specimens, the more commonly used method is that of shearing a single joint specimen under multiple (typically three) successive normal stresses. This is generally referred to as the multistage direct shear test. The primary flaw in the current multistage direct shear test procedure is that it dictates that for each stage, the specimen is sheared until satisfactory 'residual' (post-peak) shear strength is achieved [1]. To accomplish this, it may be necessary to subject the specimen to a relatively large shear displacement, often as much as several centimeters. The large shear displacement causes a significant amount of damage to the small scale asperity profile of that specimen. The specimen is then typically unloaded and returned to its original position (pre-shear displacement) to be sheared under an increased normal stress [1, 2], although the final position of each stage can be used as the starting point for the shear displacement for the next stage [2].

Figure 1 displays an idealized shear stress vs. shear displacement graph corresponding to a direct shear test performed in accordance with the 'standard' multistage procedure, in which the large specimen displacements associated with this testing method are evident.

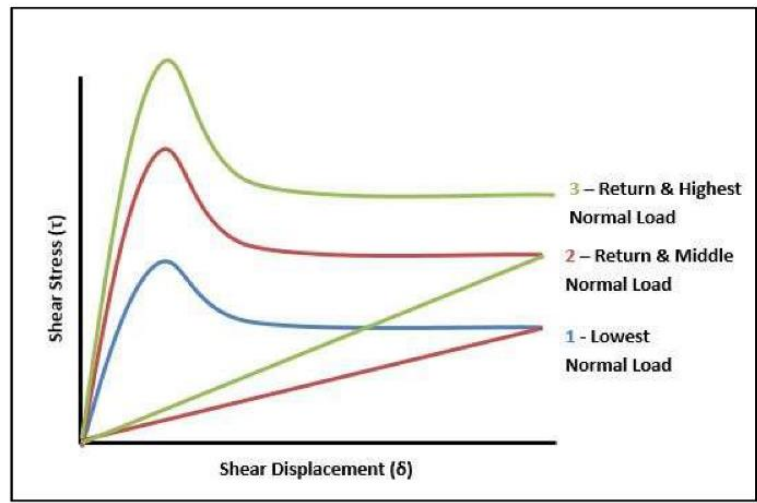

Fig. 1. Typical (idealized) test data associated with the 'standard' multistage direct shear test procedure displaying the large displacements by which the specimen is sheared before being returned to its original position. [10]

\subsection{Limited displacement multistage direct shear (LDMDS) test}

The limited displacement multi-stage direct shear (LDMDS) test method was developed by Bro [6] to address the testing-induced damage associated with the 'standard' multistage direct shear test procedure. This method is designed to minimize the damage caused to the surface asperities by limiting the total amount of displacement a specimen is subjected to prior to achieving the peak shear stress under each normal stress. The postpeak strength may be evaluated by performing three or more additional test stages, each involving subjecting the specimen to large displacements under a different value of normal stress. Figure 2 is an example of an idealized shear stress vs. shear displacement graph for a direct shear test performed in accordance with the LDMDS test method. It should be noted Figure 2 is not to scale and typically the peak shear stress range is only approximately 0.3 to $0.5 \mathrm{~mm}$, while the post-peak range can be 5 to $7 \mathrm{~mm}$ (or more) depending on the specimen.

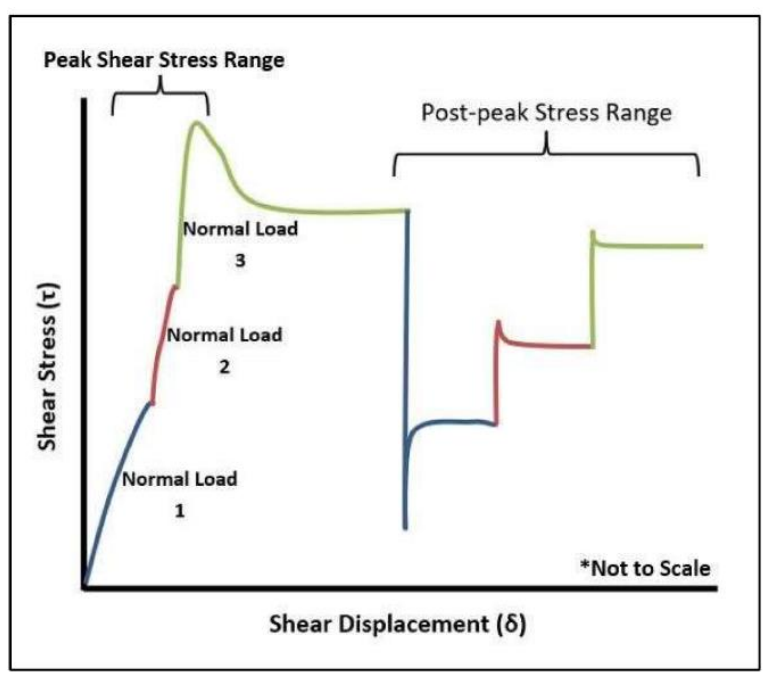

Fig. 2. Example shear stress vs. shear displacement data (idealized) from the LDMDS direct shear test. [10] 


\section{Experimental studies and results}

The overall goal of this research is to establish the accuracy of the LDMDS test method for rock joints of varying roughness as compared to ASTM (referred to as "standard") test procedures. Several experimental studies were developed and undertaken to achieve this goal. The studies involved creating replicates of rock joint specimens to perform different versions of the direct shear test on specimens with identical geometry and roughness, allowing direct comparison of the results.

To create the replicates, molds of the original real rock joint specimens were made using ReoFlex 40, a midweight liquid urethane rubber. Multiple identical replicates were then cast using synthetic materials (Figure 3 ), including high early strength Portland cement and SulfaSet bolt anchor cement. Unconfined compressive tests, nondestructive ultrasonic velocity tests, and desktop laser scans were employed for quality control of the replicates.

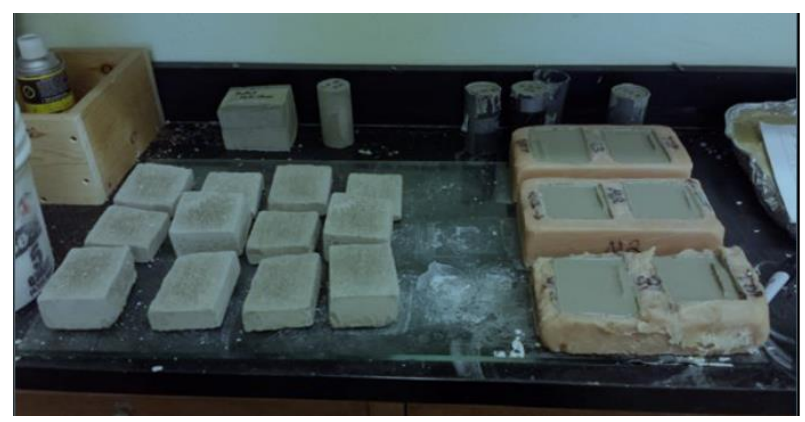

Fig. 3. Specimen molds and replicates.

The 'true' strength parameters of the undamaged specimens were identified using 'single-stage' direct shear tests, in which three specimens were subjected to large displacement under three different normal stress values. The measured peak shear strength values corresponding to three different normal stress values were combined to form the Mohr-Coulomb failure envelope, yielding the 'true' undamaged friction angle and joint shear intercept. These are also referred to as the 'baseline' values of the strength parameters. The accuracy of the 'standard' multistage and LDMDS procedures were then established by comparing them to the 'baseline' values. All of the tests (the single stage tests at each of the three normal stress values, the 'standard' multistage tests, and the LDMDS tests) were performed using a GCTS RDS200 servo-controlled direct shear system using a shear displacement rate of 0.1 to $0.2 \mathrm{~mm} / \mathrm{min}$, and were repeated multiple times.

Low values of normal stress were selected for the tests, based on the assumption that the specimens would incur less damage compared to tests performed with higher normal stress values. It follows that these conditions would be the best test of the alternative procedures: if the LDMDS procedure provides more accurate results than the standard procedure under low normal stresses, its performance under higher normal stresses is expected to be equally superior, if not even more so. Rock joint specimens with moderate to low roughness profiles, corresponding to joint roughness coefficient (JRC) values ranging from 0 to 14 [11], were selected to establish the efficacy of the LDMDS procedure over a fairly wide range of roughness values.

The main difficulty with using the LDMDS test method is identifying the exact point of shear failure and pausing the shearing stage quickly enough to prevent shearing past the first critical asperity on the surface. The following sections describe studies done by two Montana Tech master's students. The studies vary in terms of the rock joint specimens and molding materials used, and also with how the difficulty in identifying the point of shear failure was addressed.

\subsection{Phase 1: initial granitic specimens, JRC $\approx 8$}

The first phase of this research was initiated in 2014 and conducted by Montana Tech master's student Katherine Berry [8]. Five rectangular specimens of moderate roughness (JRC $\approx 8$ ) were produced from a single granitic rock containing two natural joints. The specimens were nominally 6-8 cm x 8-11 cm in size. Replicates were cast out of high early strength Portland cement and subjected to direct shear tests using a shear displacement rate of 0.1 $\mathrm{mm} / \mathrm{min}$ and normal stress values of 90,180 , and $270 \mathrm{kPa}$. Upon establishing that reasonable precision could be achieved with the single-stage tests on the replicates as shown in Figure 4, the most challenging specimen in terms of achieving repeatable stress-strain data (specimen M2) was selected for further study.

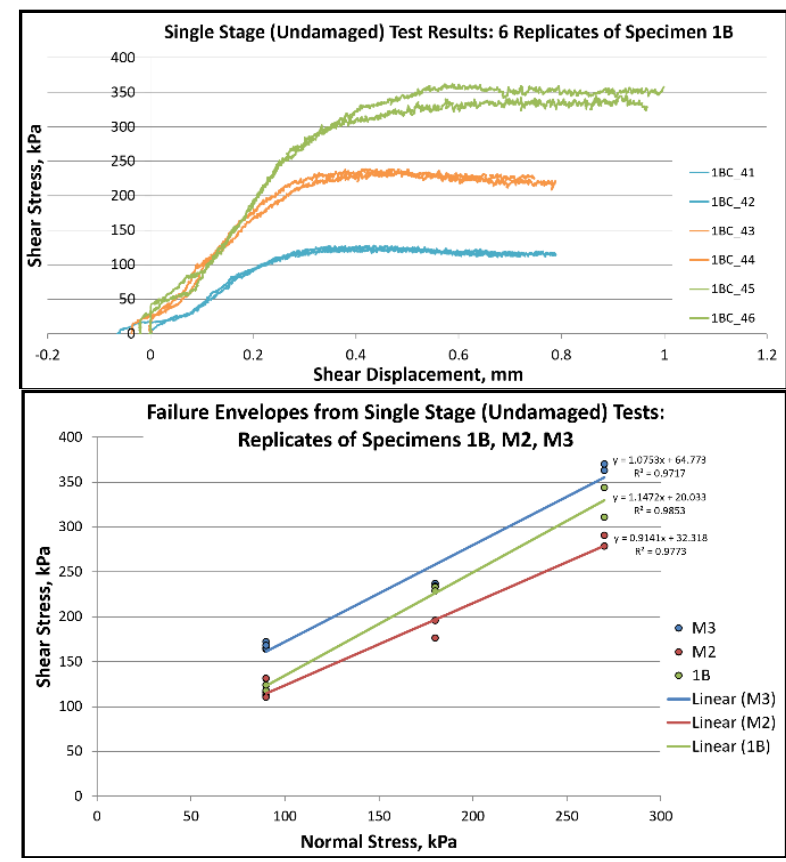

Fig. 4. Results of single-stage tests performed on cement replicates of three granitic joint specimens (1B, M2, and M3) to reveal the 'true' undamaged strength parameters. Top: the shear stress vs. shear displacement plots of 6 single-stage tests performed on 6 replicates of specimen 1B at three values of normal stress. Bottom: a plot of the normal stress vs. peak shear stress data for the undamaged tests on the specimens with linear Mohr-Coulomb failure envelopes superimposed. 
The results of the basic LDMDS procedure perfomed on replicates of specimen M2 are displayed in Figure 5. Several alternatives to the basic procedure were investigated in an effort to address the difficulty in pausing each stage at the exact point of shear failure, without displacing past the first critical asperity on the joint surface. While the LDMDS shear strength values associated with the first two stages consistently matched the shear strength of the undamaged surfaces, the shear strength associated with the third stage did not. Among the alternatives investigated, the approach of reversing the shear displacement slightly after the second shear stage, accomplished by 'returning' the specimen to a displacement value of $0.05 \mathrm{~mm}$, yielded results that best matched the undamaged strength values. Figure 6 shows the results of this version of the LDMDS test (dubbed the 'single-return' LDMDS test) performed on the real rock joint specimen $1 \mathrm{~B}$ and three of its replicates.

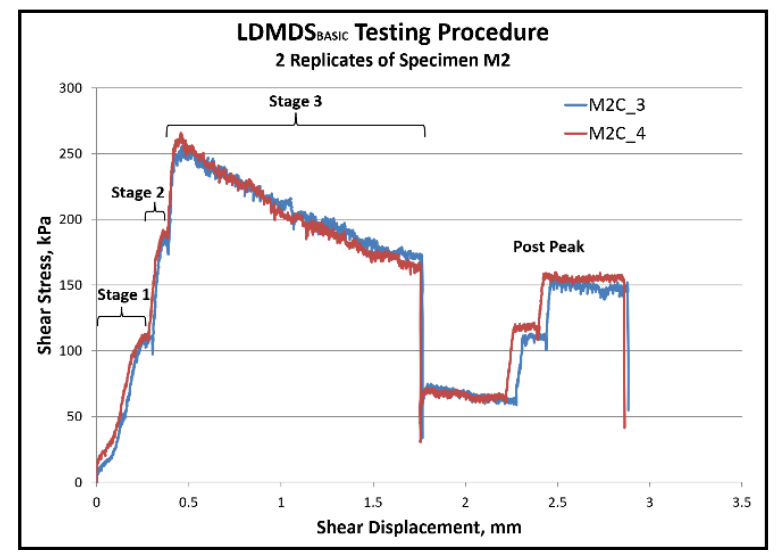

Fig. 5. Shear stress vs. shear displacement plots for two basic LDMDS tests performed on replicates of specimen M2.

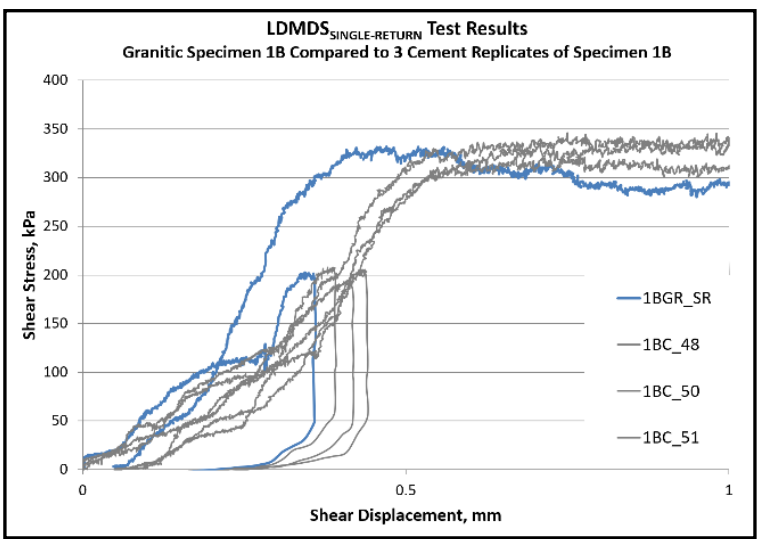

Fig. 6. Shear stress vs. shear displacement plots for the singlereturn LDMDS tests performed on specimen 1B and three of its replicates.

After testing more than 125 replicates and one of the real rock joint specimens (1B), three of the remaining rock joint specimens (M1 through M3) were used to establish the Mohr-Coulomb parameters for the undamaged and damaged surfaces that were then compared to the results of the single-return LDMDS test performed on specimen M4. The results are displayed in Figure 7. As shown in the figure, the friction angle provided by the LDMDS procedure $\left(55^{\circ}\right)$ is much closer to the 'true' undamaged friction angle $\left(53^{\circ}\right)$. The 'standard' test severely underpredicts the friction angle $\left(43^{\circ}\right)$ and overpredicts the joint shear intercept.

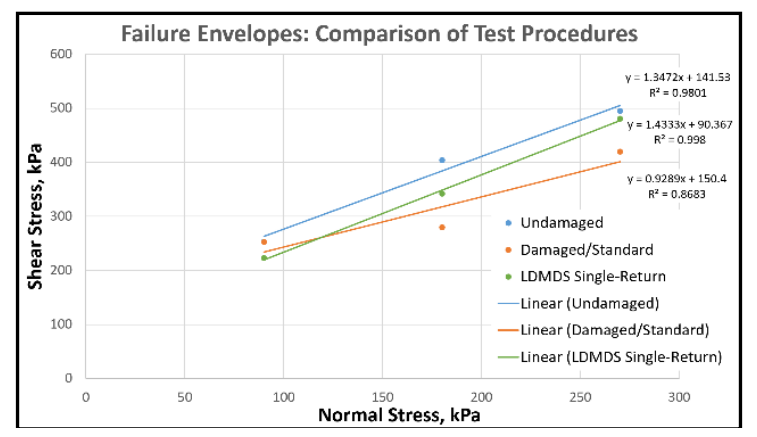

Fig. 7. Plot of the normal stress vs. peak shear stress data for four different granitic rock joint specimens (M1, M2, M3, and M4) with linear Mohr-Coulomb failure envelopes superimposed, comparing the results of the tests on 'undamaged' and 'damaged' surfaces to those of the singlereturn LDMDS test.

\subsection{Phase 2: granitic specimen $M 3, J R C \approx 8-10$}

The second phase of this research was performed in 201617 by Montana Tech master's student Michael Petro [9]. He conducted further investigation with the wellinterlocking unweathered granitic specimen M3 (JRC $\approx 8$ 10) from the previous study by K. Berry, shown in Figure 8. Using Berry's mold, additional replicates were cast out of high early strength Portland cement and subjected to direct shear tests using the same normal stress values.

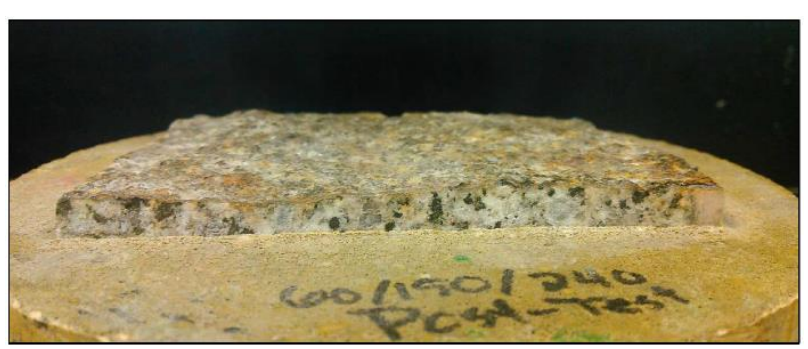

Fig. 8. Photograph of specimen M3 (JRC $\approx 8-10)$ showing its roughness profile. For scale, the specimen is $110 \mathrm{~mm}$ long by $85 \mathrm{~mm}$ wide.

The peak shear strengths identified for the 'undamaged' single-stage tests were in good agreement $(12 \%)$ with each other. The average values were in excellent agreement with the previous results (4\%) with the exception of the results associated with the lowest normal stress: the average shear strength identified in the Phase 2 study was 20\% lower than in Phase 1, attributed to slightly different specimen positioning by the operator exacerbated by the testing machine operating at the very low end of its range. While the discrepancy in the shear strength associated with the lowest normal stress caused the friction angle for the undamaged M3 surfaces to be higher in the Phase 2 study $\left(51^{\circ}\right)$ than in the Phase 1 study $\left(47^{\circ}\right)$, the values were nevertheless quite close.

Three standard tests and three basic LDMDS tests (with no 'return' reverse shear displacement stage) were 
performed on the replicates. The results are displayed in Figure 9. The top and middle graphs display the shear stress vs. shear displacement plots for the standard and LDMDS tests, respectively; for clarity, only the data associated with the 'peak' shear strengths are shown for the LDMDS tests. The bottom graph shows the best fit linear Mohr-Coulomb failure envelopes overlying the normal stress vs. peak shear stress data for the three types of tests. Visually evident in the bottom graph, this study showed an even more pronounced agreement between the friction angles interpreted from the LDMDS test $\left(45^{\circ}\right)$ and the 'true' undamaged single-stage tests $\left(51^{\circ}\right)$, compared with the standard test, which significantly underestimated the friction angle $\left(22^{\circ}\right)$ because of the damage done to the specimen prior to the second and third shear stages.
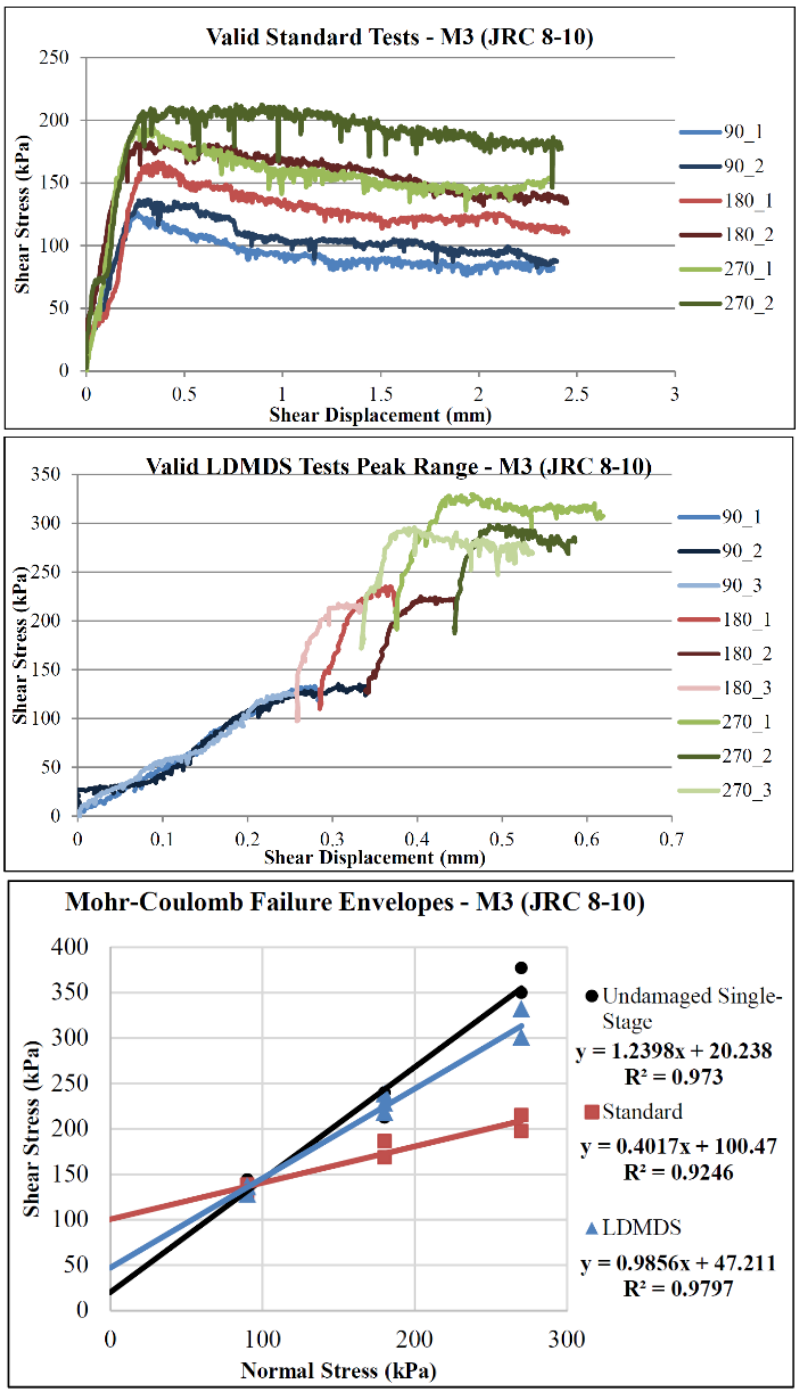

Fig. 9. Direct shear test results for cement replicates of specimen M3. Top: shear stress vs. shear displacement plots for the standard tests. Middle: Shear stress vs. shear displacement plots for peak stress region of the LDMDS tests. Bottom: plot of the normal stress vs. peak shear stress data, with linear Mohr-Coulomb failure envelopes superimposed, comparing the results of the tests on the 'undamaged' surfaces in the single-stage tests and 'damaged' surfaces in the standard tests to those of the basic LDMDS tests.

\subsection{Phase 3: additional specimens, JRC $\approx 0-14$}

The focus of the third phase of the study, continued by M. Petro in 2017-18 [10], was to expand the range of JRC values investigated. Three new joint specimens were selected: 1) a slightly metamorphosed, unweathered bedding plane in a laminar siltite containing submillimeter asperities (JRC $\approx 0-2), 2$ ) a joint surface with evenly distributed millimeter-scale asperities (JRC $\approx 4-6$ ) in a moderately weathered anorthosite, and 3) a joint in a moderately weathered anorthosite with evenly distributed millimeter-scale asperities on a surface that undulates with an amplitude of 2 to $3 \mathrm{~mm}$ (JRC $\approx 12-14)$. These specimens, labeled M4, M5, and M6, respectively, were smaller than than the specimens used in the first two phases of the research and ellipsoid in shape, as shown in Figure 10, because they were selected from available rock core. (Note that specimen M4 in this phase of the research is entirely different from the specimen M4 used in phases 1 and 2.)

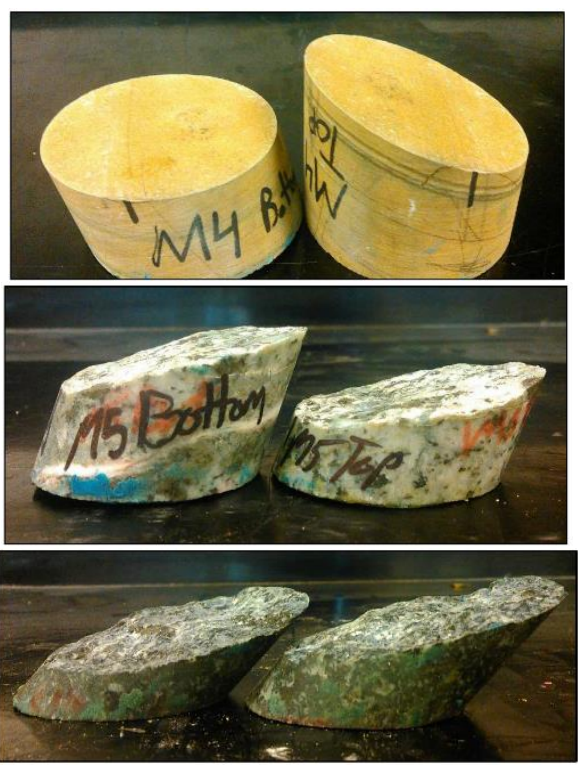

Fig. 10. Photographs of specimens M4 with JRC $\approx 0-2$ (top), M5 with JRC $\approx 4-6$ (middle), and M6 JRC $\approx 12-14$ (bottom), showing their roughness profiles. For scale, the diameters of the specimens are $60 \mathrm{~mm}$ (top) and $47 \mathrm{~mm}$ (middle and bottom).

Molds were made and replicates were cast out of SulfaSet bolt anchor cement. As in the second phase, direct shear tests were performed on the specimens using the 'basic' version of the LDMDS test, without using reverse shear displacement to 'return' the specimen to a smaller value of shear offset, but higher normal stress values $(200,400$, and $600 \mathrm{kPa})$.

The shear stress vs. shear displacement graphs are not included in this paper due to space constraints; they are available online through Montana Tech's Digital Commons [10]. Figure 11 contains the normal stress vs. shear stress plots with Mohr-Coulomb failure envelopes superimposed for the cement replicates of specimens M4 (top graph), M5 (middle graph), and M6 (bottom graph). 

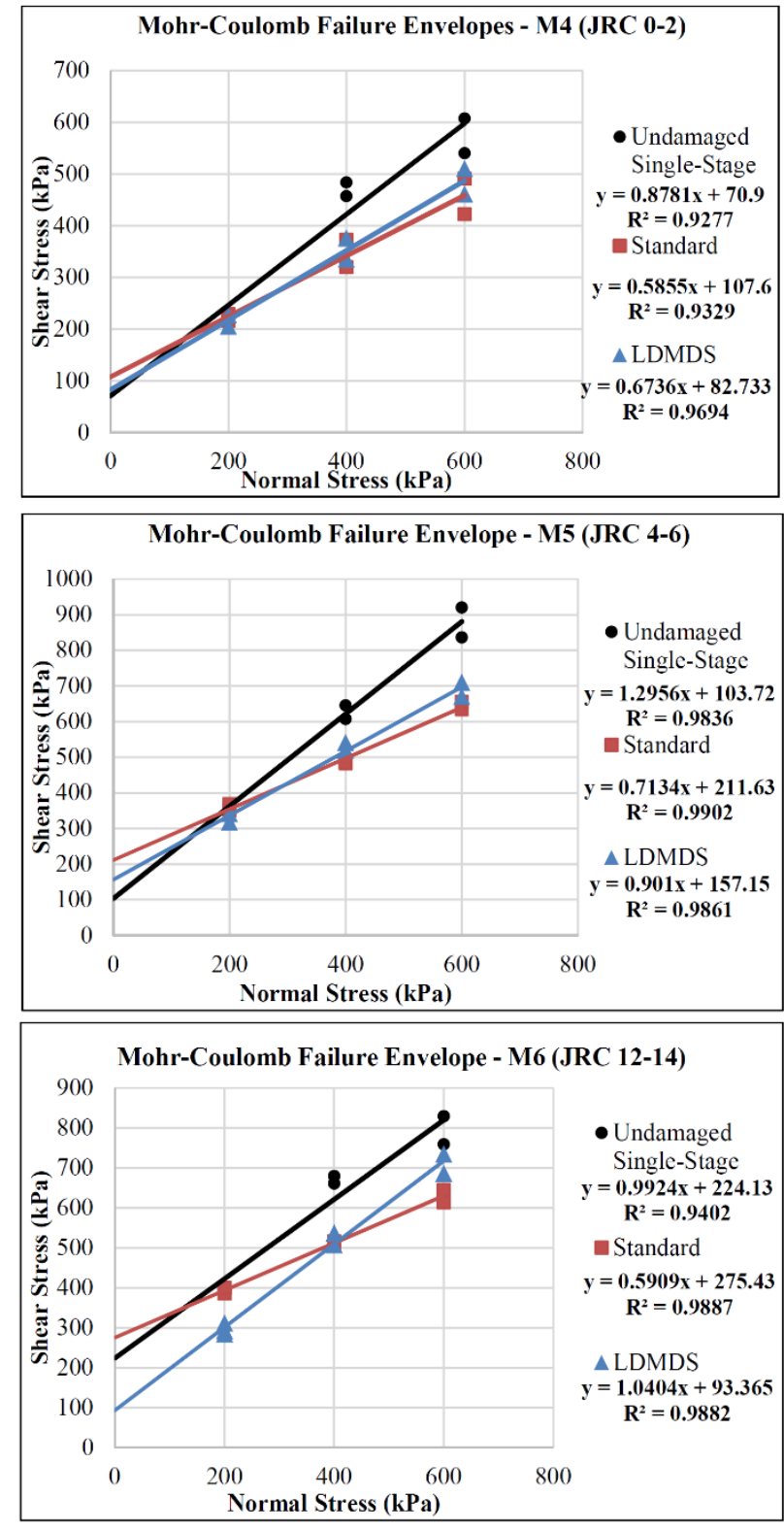

Fig. 11. Plot of the normal stress vs. peak shear stress data for cement replicates of three different rock joint specimens: M4 JRC $\approx 0-2$ (top), M5 JRC $\approx 4-6$ (middle), and M6 JRC $\approx 12-14$ (bottom) with Mohr-Coulomb failure envelopes superimposed.

Although the Phase 3 LDMDS tests technically do provide friction angle values that are closer to the 'undamaged' values than those provided by the standard tests, it is evident that the superiority of the procedure is not as pronounced for the two smoother specimens (M4 and M5). There are several possible explanations related to both changes that were made for the Phase 3 experiments and the inherently different behavior of smoother joint surfaces; further investigation is being conducted.

\section{Summary and conclusions}

The LDMDS test is proposed as an alternative to the current standard multistage direct shear test used for rock joints, which involves successive shearing of the specimen to large displacments, damaging its surface asperities and consequently resulting in underestimation of friction angle and overestimation of joint shear intercept. The LDMDS test minimizes asperity damage by immediately pausing shear displacement once peak shear stress has been reached, then proceeding to shear the specimen under the following normal stress value, and shearing into the post-peak region only after identifying multiple values of peak shear strength.

An experimental study was conducted to validate the LDMDS test procedure, using replicates of rock joint surfaces cast out of cement to provide specimens with identical joint surfaces to allow direct comparison of the results; real rock joints were also tested. The results of the LDMDS tests were compared to the 'undamaged' strength of the surfaces, as established via single-stage direct shear tests on the replicates, under normal stresses ranging from 90 to $600 \mathrm{kPa}$. The most challenging aspect of the LDMDS procedure is the difficulty in identifying and immediately pausing the test at the exact point of shear failure. The tests performed in this study show that while the standard direct shear test procedure, as anticipated, resulted in underestimation of the friction angle and overestimation of the joint shear intercept, the LDMDS test procedure is able to provide more accurate peak shear strength parameters for strong rock joints at low normal stresses, facilitating safe design without being overly conservative. As anticipated, the LDMDS procedure showed more significant improvement for tests performed on rougher specimens. The results demonstrate that this approach provides a potentially valid alternative to the current standard test procedures.

\section{References}

1. ASTM, ASTM D5607-16 (ASTM, 2016)

2. R. Ulusay, The ISRM Suggested Methods for Rock Characterization, Testing and Monitoring: 2007-2014 (Springer, 2014)

3. M.J.A. Leal-Gomes, C. Dinis-Da-Gama, 11th ISRM Congress (2007)

4. Q. Liu, Y. Tian, D. Liu, Y. Jiang, Eng. Geol., 228, 282-300 (2017)

5. N. Barton, J. of Rock Mech. and Geot. Eng. 5, 4, 249-261 (2013)

6. A. Bro, personal communication, (August 2013, September 2014 and March 2017)

7. S. Warren (NIOSH Spokane Research Center), personal communication (January 2018)

8. K. Berry, M. McCormick, S. Berry, S. Adams, M. MacLaughlin, Evaluation of the Continuous Multistage Direct Shear Test Procedure for Rock Joints (Unpublished master's research paper, Montana Tech, 2015)

9. M. Petro, B. Sordo, S. Berry, M. MacLaughlin, K. Berry, 51st US Rock Mechanics/ Geomechanics Symposium Paper 17-946 (2017)

10. M. Petro, (Montana Tech master's thesis 2018) available: digitalcommons.mtech.edu/grad_rsch

11. N. Barton, V.Choubey, Rock mechanics 10, 1-2, 1-54 (1977) 